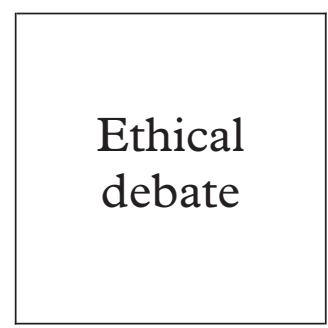

Correspondence to:

Dr A J Winter, Department of Genitourinary Medicine, Royal Infirmary, Glasgow G31 2ER.

Accepted for publication 7 July 1999

\title{
Is partner notification in the public interest?
}

\author{
Coordinated by Andrew J Winter
}

\section{Introduction}

Partner notification for HIV infection is a systematic way of notifying the contacts of an HIV infected individual who may themselves be at risk of HIV. The "index case" must normally give consent for this. A team from Birmingham describes the dilemma they faced in trying to identify sexual contacts of a patient who died shortly after being informed of her HIV diagnosis, and who was therefore unable to give such consent. We invited comments from a healthcare ethicist and the Terrence Higgins Trust (THT), and summarise advice given by the UK General Medical Council (GMC), the UK Department of Health (DoH), and the Medical Protection Society (MPS).

\section{Who is at risk?}

\author{
Andrew J Winter, David Mullis, Keith W Radcliffe
}

The index case was a woman in her 30s who first attended our clinic with extensive atypical vulval herpes. At that time we suspected underlying immunodeficiency but she declined HIV testing and failed to attend two follow up appointments. Four months later she reattended with extensive recurrent herpes, $12 \mathrm{~kg}$ weight loss, fever, intractable nausea, and malaise. She disclosed that oesophageal candidiasis had been diagnosed 2 days earlier in another hospital, and with her permission we confirmed this. She accepted co-trimoxazole for prophylaxis of Pneumocystis carinii pneumonia, and agreed that we inform her general practitioner that we suspected a diagnosis of AIDS. However, she declined HIV testing and then failed to reattend or even let her general practitioner see her. Her condition deteriorated and she was finally admitted to a local hospital where she agreed to an HIV test. Sadly she died of cryptococcal disease 12 hours after being given the news that she was indeed HIV seropositive.
According to the ward staff, the patient had confided in no one except a close friend, who declined an interview with a health adviser but agreed to talk to the patient's general practitioner. The friend alleged that the deceased had had many sexual partners in the past few years. The patient's family also appeared to be aware of the true diagnosis but we were uncertain if the patient had freely informed them, or if they were making an educated guess. The position was complicated by our not having had clinical responsibility for the patient in her final illness.

Our dilemma was whether we could enlist the patient's close family or friends in an attempt to identify putative sexual contacts who could be at significant risk of HIV infection, and how sure we had to be about the alleged relationship before actually inviting an individual to attend to discuss HIV testing.

\section{What is in the public interest?}

\section{Heather Draper}

The General Medical Council notes that the principal justification for disclosing confidential information in this case is the public interest. However, two competing views of the public interest are at play here. The first is that the public interest is best served by finding and
University of
Birmingham, Birmingham B15 2TT $\mathrm{H}$ Draper, lecturer in biomedical ethics treating as many HIV infected individuals as possible. The second is that the public interest is better served by defending confidential testing and treatment of those with HIV presuming, firstly, that individuals may be deterred from testing if confidentiality is not guaranteed 
and, secondly, that individuals who test positive will take steps to avoid infecting others. Thus, the ethical debate regarding disclosure of HIV status is not simply between the rights of the individual to confidentiality and the interest of some member(s) of the public to treatment or protection; it is a tension between two competing views of the public interest. Determining the public interest turns, or so it seems, on whether disclosure or non-disclosure is the best way of minimising further cases of HIV infection. Different countries and cultures have chosen different paths here, but in the United Kingdom the emphasis has largely been to guard confidentiality.

In the case before us, the claim of potentially infected parties to know is strengthened by the fact that the index case is dead. While confidentiality does extend beyond death, it cannot extend absolute privacy for the dead when it only extends relative privacy for the living. It is also possible to argue that the interests of the dead should come after the interests of the living.

The second issue the team considered was how certain they should be that a sexual relationship had occurred before contacting the individual concerned. For the sake of argument, I will assume that this was a continuation of their concerns about breaking confidentiality. Although it may be argued that the putative contacts have an interest in knowing that they may have been infected, it is less clear that they need to know the source of their infection. If it is possible to persuade them to come in for testing without telling them about an individual's HIV status, a breach of confidentiality is not warranted. We are then left with two moot points. Firstly, have their interests been sufficiently met if they are warned but refuse to take the warning seriously because no name is supplied? Secondly, if they do not take this warning seriously enough to be tested, will they take it seriously enough to protect others from infection? The first question raises issues about the quality of information needed to make an autonomous decision, and the second invites consideration of whether the public interest also justifies compulsory testing and the criminalisation of knowingly infecting an individual with HIV.

In conclusion, an argument can be made for approaching the family and potential sexual contacts in this case. The team needs to consider not only whether they are justified in breaching confidentiality but also to whom confidential information is to be disclosed. Disclosure to family members is only justified to those likely to be able to help in partner notification. The duty to the putative contact may then be discharged by giving a warning about the risk rather than information about the source of the risk.

\section{Is there really a dilemma?}

\section{Lisa Power}

On the basis of the information given, the Terrence Higgins Trust does not view this as an ethical dilemma and we do not believe that disclosure would be warranted. Our views are based on practical, as much as ethical, grounds.

(1) There is no clarity about who, if anyone, has been at risk-the history consistently uses phrases such as "alleged," "appeared to be," "uncertain," and "putative."

(2) Even were all these possibilities to be actualities, there are likely to have been a number of people at risk who could not easily be traced. This is not an unusual situation, and we would question why this particular woman's case has been singled out for this kind of pressure. While we agree on the importance of reaching people who have been at risk, we do not believe that contact tracing is likely to be the most effective response in this generalised situation. Rather, education about HIV, risk, and sexual health checks should be targeted at the relevant group(s) within the local community.
(3) There are, however, clear lessons about the need for a proper logical and transparent disclosure policy on the part of the clinic and a strong relationship with those local agencies which can target health promotion work to relevant communities at significant risk. We would argue that such organisations, in alliance with local clinicians, are best placed to reach both those who have been at risk and those who may be in future, and to work within local communities to improve understanding of sexual health.

(4) On a further practical note, has the clinic considered that, if a clear refusal to follow the wishes of a patient became widespread knowledge locally, other people in that community would have logical grounds to refuse to attend this clinic or any other? We deal regularly with people, particularly those from marginalised communities, who are reluctant to access services they need because of strong fears about loss of privacy in attending STI or GUM clinics. Confirmation of those fears would, in the long run, be counterproductive for public health.
Trust, London WC1X 8JU

L Power, assistant director of health promotion 


\title{
What advice did we get at the time?
}

\author{
Andrew J Winter, Keith W Radcliffe
}

Advice in general terms was sought from the UK General Medical Council, from the UK Department of Health (DoH), and from one of the national medical defence organisations, the Medical Protection Society (MPS).

\section{UK General Medical Council}

The GMC reminded us that doctors have a general obligation to confidentiality which extends beyond death. However, disclosure may be justified to protect a person from a risk of death or serious harm. Specific guidance is found in paragraph 22 of Serious Communicable Diseases:

You may disclose information to a known sexual contact of a patient with HIV where you have reason to think that the patient has not informed that person, and cannot be persuaded to do so. In such circumstances you should tell the patient before you make the disclosure and be prepared to justify a decision to disclose information.

In this specific case the GMC understood that we would have to judge carefully the reliability of any evidence regarding the risk to a third party. If we were reasonably satisfied of a risk of HIV transmission, then we could disclose information as necessary provided we could justify our actions. We should discuss the case with colleagues, and carefully document all actions and discussions. This did not help us decide how (if at all) we were to trace sexual contacts.

\section{Department of Health}

A senior medical officer at the $\mathrm{DoH}$, when asked to advise, reiterated the GMC's guidance. Her interpretation of the GMC's guid-

ance in these circumstances was that if a former sexual partner could reliably be identified, such a person might be considered at "risk of serious harm" if HIV infected and not identified as such. Such a person should be given the opportunity to benefit from optimal medical management of their condition and thus be in a position to prevent onward transmission of HIV to current or future sexual partners, protecting them also from "risk of death and serious harm."

\section{UK Medical Protection Society (MPS)}

The MPS reminded us that a doctor's duty of confidentiality has been held to extend beyond the grave. If identifiable individuals were thought to be at significant risk of HIV infection then this could constitute grounds for breaching confidentiality, not least in order for such individuals to avail themselves of appropriate treatment. The patient's general practitioner could be approached to assist in such identification. However, it would not be appropriate to disclose information to relatives who were not at personal risk simply because they wished to know the cause of death. The society was concerned that the patient's persistent refusal to have an HIV test strongly suggested that she did not wish the diagnosis made known, even though her specific wishes regarding disclosure were unknown. The reasons for any disclosure should be carefully documented in contemporaneous records (as is good medical practice) so that we could justify our actions in the unlikely event of a complaint.

1 General Medical Council. Serious communicable diseases. London: GMC, 1999.

\section{What did we do?}

\author{
Andrew J Winter, David Mullis
}

After careful consideration with colleagues, ward staff, and discussion with the patient's general practitioner, it became clear to us that at least one member of the family was fully aware of the true diagnosis. In addition, we began to see, for HIV testing, patients who named our index case as the reason for their concern as a result of rumours spreading in the community. When we interviewed the relative,

she assured us that the index case had disclosed her HIV status shortly before her death, and she helped identify additional individuals as sexual contacts. So far six individuals at known risk have attended, and all were HIV seronegative. However, we have insufficient information to contact two more individuals about whom we are seriously concerned, and the source case remains undiscovered. 


\section{Conclusion}

\author{
Andrew J Winter
}

This case illustrates poignantly the conflict between different interpretations of the "public interest." The GMC's current position leans towards limited breaches of confidentiality in the extreme example where a known person is at significant risk of death or serious harm. Disclosure is allowed, but appears not to be a duty (unlike the situation, say, regarding driving with a medical contraindication). The decision is left to the individual practitioner. Patient support groups such as the Terrence Higgins Trust remain concerned that this position undermines trust and so prevents uptake of HIV testing. We are convinced that sensitive and appropriate partner notification should be an integral part of HIV case management. Our case shows how fragmented care and a frightened patient can undermine any attempt to address this important issue, not least by preventing the timely medical intervention which may have saved her life.

We are grateful to Dr Susan Turnbull, senior medical officer, Department of Health; Dr Mark Dudley, medicolegal adviser, Medical Protection Society; and Mr Neil Marshall of the GMC, for their advice.

This case was presented for discussion at the Midlands HIV Interest Group, September 1998, and we thank participants for their contribution. 